\section{On the Origin of Early Superhumps in WZ Sge-Type Stars}

\begin{tabular}{|c|c|}
\hline \multicolumn{2}{|c}{ Taichi KATO } \\
\hline Sakyo-ku, Kyoto 606-8502
\end{tabular}

tkato@kusastro.kyoto-u.ac.jp

\begin{abstract}
The origin of early superhumps, which are double-wave

periodic modulations seen only during the earliest stage of WZ Sge-type outburst, has not been well understood. Based on recent discovery of two-armed arch-like patterns on Doppler tomograms in conjunction with early superhumps, we propose a new interpretation on the origin of early superhumps, following the new interpretation by Smak (2001) and Ogilvie (2001) of the twoarmed pattern seen in IP Peg. If we consider irradiation of the elevated surface of the accretion disk caused by vertical tidal deformation, we can consistently explain the observed features on Doppler tomograms and photometric waves at the same time. We interpret that a combination of low mass-ratios $(q)$ and low masstransfer rates, necessary to give rise to these tidal effects, discriminate WZ Sge-type stars from other SU UMa-type dwarf novae. Based on recent fluid calculations, such an effect would be observable in higher $q$ systems. We interpret that RZ Leo is an example of such objects.
\end{abstract}

Key Words: accretion, accretion disks — stars: novae, cataclysmic variables - stars: dwarf novae stars: individual (WZ Sagittae, RZ Leonis)

\section{Introduction}

Dwarf novae are a class of cataclysmic variables (CVs), which are close binary systems consisting of a white dwarf and a red dwarf secondary transferring matter via the Roche-lobe overflow. WZ Sge-type dwarf novae (cf. Bailey (1979); Downes, Margon (1981); Patterson et al. (1981); Downes (1990); O'Donoghue et al. (1991); Kato et al. (2001b)) are a small subgroup of dwarf novae characterized by the long $(\sim 10$ yr) outburst recurrence time and the large $(\sim 8 \mathrm{mag})$ outburst amplitude. They are a subclass of SU UMa-type dwarf novae (cf. Warner (1995b).

The most remarkable signature of WZ Sge-type outbursts is the presence of "early superhumps" during their earliest stage of superoutbursts. Early superhumps have a period extremely close to that of the bi-

1 This feature is also referred to as orbital superhumps (Kato et al., 1996) or outburst orbital hump (Patterson et al., 1998). nary period ${ }^{2}$, and commonly show double-humped profile (figure 1; see also Kato et al. (1996) and Kato et al. (1998) for detailed discussions), in contrast to ordinary superhumps of SU UMa-type dwarf novae [see Warner (1995b) for basic properties of SU UMa-type dwarf novae]. Early superhumps are the most discriminative feature of WZ Sge-type outbursts, and have not been detected in other dwarf novae (see Kato et al. (2001a) and Kato et al. (2001b)). Although several models have been historically proposed [e.g. enhanced hot spot (Patterson et al., 1981), immature form of superhumps (Kato et al., 1996), jet or thickened disk (Nogami et al., 1997)], none of them has successfully explained all the observed features of early superhumps.

\section{Recent Observations of Early Superhumps}

During the early stage of the 2001 outburst of WZ Sge (e.g. Ishioka et al. (2002)), several authors Steeghs et al. (2001); Baba et al. (2001)) detected two-armed arch-like structures on Doppler tomograms, Marsh, Horne, 1988) of HeII and CIII/NIII emission lines. The feature resembled those of 'spiral structure' seen on Doppler tomograms of the dwarf nova IP Peg Steeghs et al. (1997); Steeghs et al. (1998)) in outburst. The spiral structure in Doppler tomograms of IP Peg has been widely believed to represent tidally induced spiral shocks or spiral waves (Steeghs et al., 1997). However, it is not clear whether the same interpretation can apply to a completely different (very low mass-ratio, $q=M_{2} / M_{1}<0.1$ in contrast to $q \sim 0.5$ in IP Peg, and very low-mass transfer rate, several orders of magnitudes lower than that of IP Peg) binary parameters of WZ Sge.

At the epoch of the detection of two-armed archlike structures in WZ Sge, the star showed double-wave early superhumps (Ishioka et al., 2002). Although there have been suggestions that early superhumps in photometry and two-armed structures on Doppler tomograms have the same origin (Ishioka et al. (2002); Baba et al. (2002); Kuulkers et al. (2002)), no promising idea has been proposed to explain these features in the same scheme.

${ }^{2}$ The best-established case is the 2001 superoutburst of WZ Sge (Ishioka et al. (2002 and references therein). In a few other systems (e.g. AL Com: Kato et al., (1996), Patterson et al. (1996), Nogami et al. (1997): EG Cnc: Kato et al. (1997); Matsumoto et al. (1998); Patterson et al. (1998), the periods of early superhumps have been found to be in good agreement with their quiescent photometric periods (most likely representing orbital periods). 


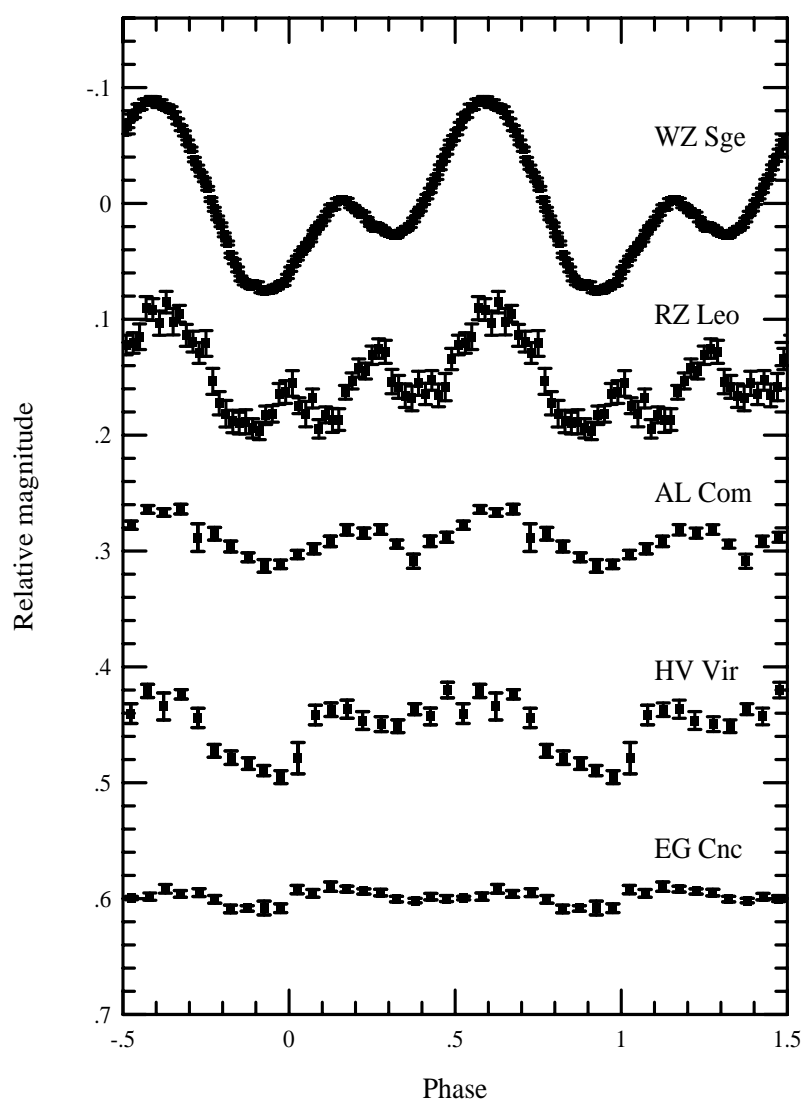

Figure 1: Comparison of profiles of early superhumps of WZ Sge-type stars. The data are taken from: WZ Sge (Ishioka et al., 2002); RZ Leo (Ishioka et al., 2001); AL Com (Kato et al., 1996); HV Vir (Kato et al., 2001b); EG Cnc (Matsumoto et al., 1998). The phase in WZ Sge corresponds to the orbital phase. Since the exact binary phases are unknown for the rest of the objects, the phases were arbitrarily taken so that the main hump maxima best match that of WZ Sge. All stars exhibit characteristic double (or sometimes triple) humps with unequal amplitudes.

\section{Tidally Distorted Accretion Disks in Close Binaries}

Most recently, an alternative idea has been proposed to explain arch-like structures in the outbursting IP Peg disk, by considering the irradiation of the elevated disk formed by the horizontal convergence $(-\operatorname{div}(\boldsymbol{v}))$ of three-body orbits (Smak, 2001). This interpretation successfully reproduced the arch-like structures, and the observed strong intensity in the region of $\left(v_{x}>0, v_{y}>\right.$ 0 ), which is difficult to explain within the scheme of conventional tidally induced spiral shocks. Ogilvie (2001) studied tidal distortion of fluid disks, and succeeded in reproducing the basically same features. Ogilvie (2001) showed, in realistic fluid disks, that $m=2$ inner vertical resonance play a more important role even in high $q$ binaries.

\section{New Interpretation of Early Superhumps}

In low $q$ systems like WZ Sge, tidal force of the secondary is so weak on a compact disk that a condition is usually achieved that angular momentum is more effectively transported by viscosity than tidal force of the secondary (Lin, Papaloizou, 1979). However, during the early stage of vigorous outbursts of WZ Sge-type stars, the disk can sufficiently expand (Osaki, 1995). In usual SU UMa-type dwarf novae, the growth of 3:1 resonance, which is responsible of superhumps (Whitehurst (1988); Hirose, Osaki (1990); Lubow (1991)), is rapid enough to effectively truncate the disk at the resonance radius. In low $q$ WZ Sge-type stars, the slow growth [the growth rate being proportional to $q^{2}$ (Lubow, 1991)] can allow the disk to expand beyond the 3:1 resonance. 3 In such a condition, tidal distortion of the outer edge of the accretion disk, as proposed by Smak (2001) and Ogilvie (2001), can effectively work, as shown in figure $3, q=0.1$ case of Ogilvie (2001). This effect is predominant in the outer edge of the accretion disk, which can explain stronger and distorted emission lines in low velocity regions (e.g. Baba et al. (2002)). Since high-excitation emission lines like HeII require a high temperature $(\sim$ $25000 \mathrm{~K}$ ), the concentration of emission lines and archlike patterns in low velocity regions is otherwise difficult to explain (Smak, 2001). The arch-like pattern in Doppler tomograms in WZ Sge is also stronger in the region of $\left(v_{x}>0, v_{y}>0\right)$, which also can be naturally explained as a result of stronger tides near the secondary, as in Smak (2001) and Ogilvie (2001).

The maximum phases of early superhumps have

\footnotetext{
3 Some observational implications of WZ Sge-type stars have been proposed to be a consequence of the disk beyond the 3:1 resonance (Kato et al., 1998).
} 

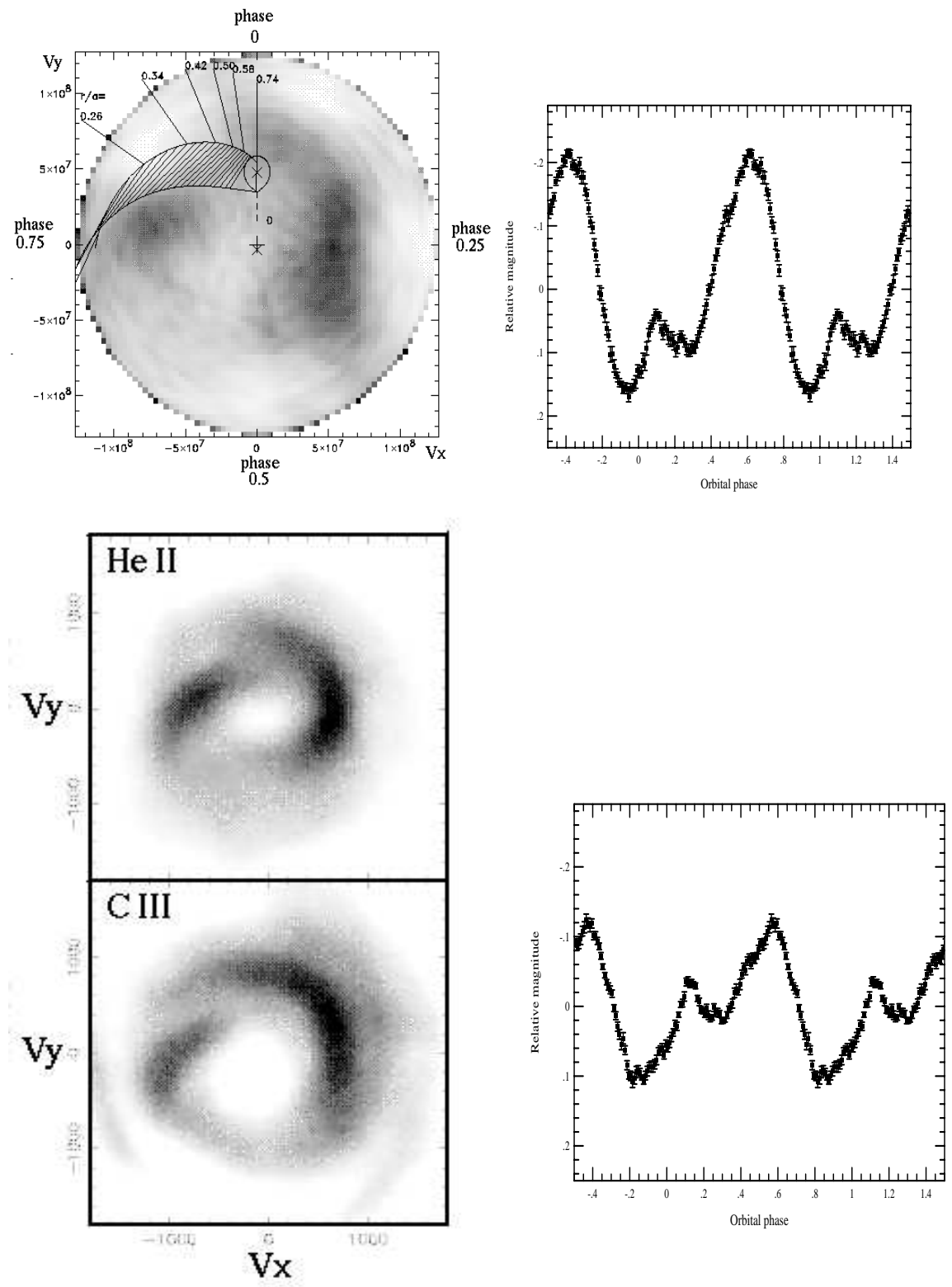

Figure 2: (Upper left) Doppler maps of HeII from Baba et al. (2002) on 2001 July 24. The Roche lobe and streamline were drawn with binary parameters of $i=75^{\circ}$ and $q=0.071$. The 'phase' values represent the direction of the observer at given orbital phases. The stronger HeII feature is seen in the region with $v_{x}>0$, i.e. facing the observer near the binary phase 0.1-0.4. (Upper right) Phase-averaged light curve of early superhumps on the same night (the data are from Ishioka et al. (2002)). The light curve shows double maxima of different amplitudes. The stronger hump is seen near the binary phase 0.6-0.7, almost opposite to what is expected from the Doppler tomogram. (Lower left) Doppler maps of HeII and CIII from Kuulkers et al. (2002) (the data were taken by D. Steeghs on 2001 July 28). Two-armed arch-like structures are clearly seen. The asymmetry of the intensities of between arches is stronger in CIII. (Lower right) Phase-averaged light curve on July 28. The main maximum moved to phase $0.5-0.6$, which is in agreement with the observed counterclockwise rotation of the features on Doppler maps since July 24. 
been also problematic. Early superhumps in WZ Sgetype dwarf novae consist of two maxima of different amplitudes (figure 1). In WZ Sge, the brighter maximum is around binary phase $0.6-0.7$ (Ishioka et al. 2002). While models involving an enhanced hot spot as in Patterson et al. (1981) would require such a maximum corresponding to the orbital phase of a stronger emission feature, the available observation shows a complete phase reversal (figure 22). Such a reversal can be naturally understood if one considers the source of emission lines and stronger continuum is an irradiated surface (Smak (2001); Ogilvie (2001)) of the accretion disk. [Since CVs are known to emit most of their energy in the UV wavelengths (e.g. Warner (1995a)), re-emission of a small fraction of intercepted UV photons in the optical wavelengths is expected to explain the observed amplitudes of continuum variation (early superhumps)]. The decrease in amplitude of early superhumps in accordance with the fading of the outburst (e.g. Ishioka et al. (2002)) can be also naturally understood as an effect of shrinkage of the disk, resulting in decreasing tidal effects.

Ishioka et al. (2002) also showed that the exact period of early superhumps is very slightly $(0.05 \%)$ shorter than the orbital period. This effect was observed as a phase-shift of maxima of early superhumps. This change looks likely to correspond to a counterclockwise rotation of the patterns on Doppler tomograms at different epochs (figure 2). The rotation of patterns may reflect the variation of the direction of the 'maximum height distortion' toward a smaller disk radius (see figure 2 of Ogilvie (2001)). Further detailed calculations of a time-evolution of the outbursting WZ Sge disk would provide a stringest test to this interpretation.

Most recently, Osaki, Meyer (2001) explained early superhumps (which they call early humps) by considering the manifestation of the tidal 2:1 resonance in accretion disks of binary systems with extremely low mass ratios. The present interpretation is different from that of Osaki, Meyer (2001) in its basic mechanism: Osaki, Meyer (2001) requires tidal dissipation while the present interpretation only requires tidal distortion of a steady flow (hence it does not require a shock). The present interpretation has an advantage over all existing models in its simplicity of the assumed physics and in that it can also explain two-armed distortion patterns on Doppler tomograms as well as photometric signals.

The reason why such features are only observed in WZ Sge-type stars can be explained as a combination of the large stored mass in quiescence (Osaki, 1995) and the delayed exertion of the 3:1 resonance, which is only reasonably achieved in extremely low $q$ and low mass-transfer rate (and presumably low quiescent viscosity) systems as in WZ Sge-type stars. Since threedimensional fluid calculation by Ogilvie (2001) showed that $m=2$ inner vertical resonance can effectively work in relatively high $q$ systems, we can expect similar phenomena expected in Lin, Papaloizou (1979) in larger $q$ systems, which cannot hold particle orbits of the 2:1 resonance within the Roche lobe. Although Osaki, Meyer (2001) also presented an idea of distinguishing WZ Sgetype stars from other CVs based on resonances involved, the present interpretation is different from that of Osaki, Meyer (2001) in that Osaki, Meyer (2001) only considered resonances in Paczynski type orbits (e.g. Ogilvie (2001)), while the present interpretation involves the three-dimensional effect as presented by Ogilvie (2001). The appearance of early superhumps and the reproduction of WZ Sge-type outburst in RZ Leo (Ishioka et al., 2001), a binary with $q=0.14$, which is approximately twice larger than those of other known WZ Sge-type stars, can be understood as one of the most distinct consequences between the present interpretation and that of Osaki, Meyer (2001).

The author is grateful to H. Baba, R. Ishioka and D. Steeghs for allowing the use of their figures. The author is also grateful to Y. Osaki for drawing attention to the paper by Lin, Papaloizou (1979). This work is partly supported by a grant-in aid (13640239) from the Japanese Ministry of Education, Culture, Sports, Science and Technology.

\section{References}

Baba, H., Sadakane, K., Norimoto, Y., Ayani, K., Ioroi, M., Matsumoto, K., Nogami, D., Makita, M., \& Kato, T. 2002, PASJ, in press (astro-ph/0112374)

Baba, H., Sadakane, K., Norimoto, Y., Nogami, D., Matsumoto, K., Makita, M., \& Kato, T. 2001, IAU Circ., 7678

Bailey, J. 1979, MNRAS, 189, 41P

Downes, R. A. 1990, AJ, 99, 339

Downes, R. A., \& Margon, B. 1981, MNRAS, 197, 35P

Hirose, M., \& Osaki, Y. 1990, PASJ, 42, 135

Ishioka, R., Kato, T., Uemura, M., Iwamatsu, H., Matsumoto, K., Stubbings, R., Mennickent, R., Billings, G. W., Kiyota, et al. 2001, PASJ, 53, 905

Ishioka, R., Uemura, M., Matsumoto, K., Ohashi, H., Kato, T., Nogami, D., Pietz, J., Martin, B., Masi, et al. 2002, A\&A, in press (astro-ph/0111432)

Kato, T., Matsumoto, K., Nogami, D., Morikawa, K., \& Kiyota, S. 2001a, PASJ, 53, 893 
Kato, T., Nogami, D., Baba, H., \& Matsumoto, K. 1998, in ASP Conf. Ser. 137, Wild Stars in the Old West, ed. S. Howell, E. Kuulkers, \& C. Woodward (San Francisco: ASP), 9

Kato, T., Nogami, D., Baba, H., Matsumoto, K., Arimoto, J., Tanabe, K., \& Ishikawa, K. 1996, PASJ, 48, L21

Kato, T., Nogami, D., Matsumoto, K., \& Baba, H. 1997, preprint, ftp://ftp.kusastro.kyotou.ac.jp/pub/vsnet/preprints/ EG_Cnc/

Kato, T., Sekine, Y., \& Hirata, R. 2001b, PASJ, 53, 1191

Kuulkers, E., Knigge, C., Steeghs, D., Wheatley, P. J., \& Long, K. S. 2002, in The Physics of Cataclysmic Variables and Related Objects, ed. B. T. Gänsicke, K. Beuermann, \& K. Reinsch (San Francisco: ASP), in press (astro-ph/0110064)

Lin, D. N. C., \& Papaloizou, J. 1979, MNRAS, 186, 799

Lubow, S. H. 1991, ApJ, 381, 259

Marsh, T. R., \& Horne, K. 1988, MNRAS, 235, 269

Matsumoto, K., Nogami, D., Kato, T., \& Baba, H. 1998, PASJ, 50, 405

Nogami, D., Kato, T., Baba, H., Matsumoto, K., Arimoto, J., Tanabe, K., \& Ishikawa, K. 1997, ApJ, 490, 840

O'Donoghue, D., Chen, A., Marang, F., Mittaz, J. P. D., Winkler, H., \& Warner, B. 1991, MNRAS, 250,363

Ogilvie, G. I. 2001, MNRAS, in press (astro$\mathrm{ph} / 0111262$ )

Osaki, Y. 1995, PASJ, 47, 47

Osaki, Y., \& Meyer, F. 2001, A\&A, in press (astro$\mathrm{ph} / 0112309)$

Patterson, J., Augusteijn, T., Harvey, D. A., Skillman, D. R., Abbott, T. M. C., \& Thorstensen, J. 1996, PASP, 108, 748

Patterson, J., Kemp, J., Skillman, D. R., Harvey, D. A., Shafter, A. W., Vanmunster, T., Jensen, L., Fried, R., Kiyota, S., Thorstensen, J. R., \& Taylor, C. J. 1998, PASP, 110, 1290

Patterson, J., McGraw, J. T., Coleman, L., \& Africano, J. L. 1981, ApJ, 248, 1067

Smak, J. I. 2001, Acta Astron., 51, 295
Steeghs, D., Harlaftis, E. T., \& Horne, K. 1997, MNRAS, 290, 28P

Steeghs, D., Harlaftis, E. T., \& Horne, K. 1998, MNRAS, 296, 463

Steeghs, D., Marsh, T., Kuulkers, E., \& Skidmore, W. 2001, IAU Circ., 7675

Warner, B. 1995a, Cataclysmic Variable Stars (Cambridge University Press, Cambridge)

Warner, B. 1995b, Ap\&SS, 226, 187

Whitehurst, R. 1988, MNRAS, 232, 35 